Notfall Rettungsmed $2011 \cdot 14: 5-5$

DOI 10.1007/s10049-010-1361-4

Online publiziert: 27. Januar 2011

(c) Springer-Verlag 2011

\author{
H.-R. Arntz ${ }^{1}$, U. Kreimeier ${ }^{2}$ \\ ${ }^{1}$ Kardiologie und Pulmonologie, Medizinische Klinik II, Charité - \\ Universitätsmedizin Berlin, Campus Benjamin Franklin, Berlin \\ ${ }^{2}$ Klinik für Anaesthesiologie, Klinikum der Universität München
}

\title{
Notfall + Rettungsmedizin ist neues Organ der agswn und DGINA
}

Liebe Leserinnen und Leser,

mit der heutigen Ausgabe der Zeitschrift Notfall + Rettungsmedizin begrüßen wir die Mitglieder der Arbeitsgemeinschaft Südwestdeutscher Notärzte (agswn) und die Deutsche Gesellschaft Interdisziplinäre Notaufnahme (DGINA) im Kreis unserer festen Abonnenten - beides ein herausragendes Ereignis für die Entwicklung unserer Zeitschrift. Diese Entscheidung der Gesellschaften für uns ist ohne Frage ein Vertrauensbeweis, der uns nicht nur zeigt, dass wir mit Notfall + Rettungsmedizin auf einem guten Weg sind, sondern ist uns auch eine Ermutigung und Herausforderung, weiter an Inhalt und Qualität der Zeitschrift zu arbeiten. Dabei wollen wir nicht verhehlen, dass wir auch auf die aktive „Mittäterschaft" des erweiterten Leserkreises zählen, sei es durch Originalien, Kasuistiken oder konzeptionelle Beiträge.

In Anbetracht der rasanten Entwicklung der Kliniklandschaft mit einer rasch zunehmenden Anzahl interdisziplinärer Notaufnahmen kommt der Schnittstelle präklinische Notfallmedizin/klinische Notfallbehandlung ohne Zweifel eine zunehmende Bedeutung zu. Die sich aus dieser Entwicklung ergebenden neuen Erkenntnisse, Probleme und v. a. Problemlösungen werden sicher in $\mathrm{Zu}$ kunft einen wesentlichen Niederschlag in unserer Zeitschrift finden. Die Bedeutung und die wissenschaftlichen Aktivitäten in der agswn herauszuheben (Stichworte: „Baden Baden“, „Leinsweiler“, „Reisensburg") hieße Eulen nach Athen tragen, denn zahlreiche der bei diesen Symposien erarbeiteten wichtigen Ergebnisse sind in den vergangenen Jahren durch Notfall + Rettungsmedizin einer breiten Öffentlichkeit vorgestellt worden. Seien Sie in diesem Sinne noch einmal auf das herzlichste willkommen geheißen. Mit einem weiteren Schritt in der Entwicklung unserer Zeitschrift möchten wir Sie ebenfalls bekannt machen.

Die Qualität einer wissenschaftlichen Fachzeitschrift wird wesentlich durch den Begutachtungsprozess für die eingereichten Manuskripte bestimmt. Notfall + Rettungsmedizin war eine der ersten deutschen Fachzeitschriften, die ein elektronisches Manuskriptbearbeitungssystem, den Editorial Manager ${ }^{\circledR}$, einführte. Autoren haben damit die Möglichkeit, in papierloser Form Manuskripte online einzureichen. Die Herausgeber können die Manuskripte „verfolgen“ und sich jederzeit über den aktuellen Bearbeitungsstand der von ihnen betreuten Arbeiten informieren. Die Redaktion kann den Manuskriptstand analysieren und z. B. Leitthemenbeiträge koordinieren. All dies könnte man unter „,workflow" zusammenfassen. Bis eine Arbeit zur Publikation angenommen ist und in der Printausgabe erscheint, vergehen aktuell 6-9 Monate. Rund 90\% aller eingereichten Manuskripte durchlaufen in dieser Zeit einen Überarbeitungsprozess, im Verlauf dessen externe Reviewer - alle Experten auf ihrem Gebiet - die Arbeit analysieren, bewerten und aus ihrer klinischwissenschaftlichen Erfahrung wichtige Aspekte aufzeigen, die vielleicht etwas mehr betont, herausgearbeitet oder ergänzt werden müssen.
Um die Transparenz des Begutachtungsprozesses und dessen Struktur zu verbessern wurde mit Beginn dieses Jahrgangs für Notfall + Rettungsmedizin eine Bewertungsmatrix entwickelt, die 12 Punkte (Items) umfasst. Hierunter fallen u. a. die wissenschaftliche Relevanz, die Verständlichkeit des Texts, die Relevanz und Aktualität der Literaturzitate, aber auch die Aussagekraft und Qualität der eingereichten Abbildungen und Tabellen. Das Ergebnis dieser Bewertungsmatrix soll als Qualitätssicherungsinstrument für den Begutachtungsprozess der eingereichten Manuskripte dienen. Wir sehen dies als weiteren Schritt, unsere Zeitschrift für Sie, liebe Leserinnen und Leser, thematisch aktuell, inhaltlich interessant, und qualitativ hochwertig zu gestalten.

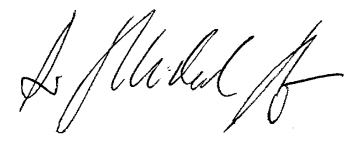

H.-R. Arntz

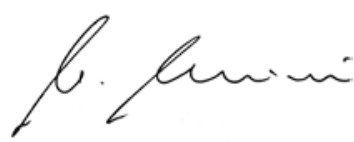

U. Kreimeier

\section{Korrespondenzadresse \\ Prof. Dr. H.-R. Arntz \\ Kardiologie und Pulmonologie, Medizinische Klinik II, Charité - Universitätsmedizin Berlin, Campus Benjamin Franklin Berlin \\ hans-richard.arntz@charite.de}

